\title{
Computed Tomography of the Abdomen without Contrast
}

National Cancer Institute

\section{Source}

National Cancer Institute. Computed Tomography of the Abdomen without Contrast. NCI Thesaurus. Code C137895.

Computed tomography of the abdomen without the use of a contrast agent. 